\title{
Influence of the exchange and correlation functional on the structure of amorphous InSb and $\mathrm{In}_{3} \mathrm{SbTe}_{2}$ compounds
}

\author{
Silvia Gabardi, ${ }^{1}$ Sebastiano Caravati, ${ }^{1, a)}$ Jan H. Los, ${ }^{2, b)}$ Thomas D. Kühne, ${ }^{2, c)}$ \\ and Marco Bernasconi ${ }^{1, d)}$ \\ ${ }^{1}$ Dipartimento di Scienza dei Materiali, Università di Milano-Bicocca, Via R. Cozzi 55, I-20125 Milano, Italy \\ ${ }^{2}$ Institute of Physical Chemistry and Center for Computational Sciences, Johannes Gutenberg University \\ Mainz, Staudinger Weg 7, D-55128 Mainz, Germany
}

(Received 4 March 2016; accepted 3 May 2016; published online 27 May 2016)

\begin{abstract}
We have investigated the structural, vibrational, and electronic properties of the amorphous phase of InSb and $\mathrm{In}_{3} \mathrm{SbTe}_{2}$ compounds of interest for applications in phase change non-volatile memories. Models of the amorphous phase have been generated by quenching from the melt by molecular dynamics simulations based on density functional theory. In particular, we have studied the dependence of the structural properties on the choice of the exchange-correlation functional. It turns out that the use of the Becke-Lee-Yang-Parr functional provides models with a much larger fraction of In atoms in a tetrahedral bonding geometry with respect to previous results obtained with the most commonly used Perdew-Becke-Ernzerhof functional. This outcome is at odd with the properties of $\mathrm{Ge}_{2} \mathrm{Sb}_{2} \mathrm{Te}_{5}$ phase change compound for which the two exchange-correlation functionals yield very similar results on the structure of the amorphous phase. Published by AIP Publishing. [http://dx.doi.org/10.1063/1.4950817]
\end{abstract}

\section{INTRODUCTION}

Phase change chalcogenide alloys are the active materials in optical data storage (digital versatile disk, DVD) ${ }^{1-3}$ and more recently they are attracting an increasing interest for several electronic applications, ranging from storage class non-volatile memories ${ }^{4-6}$ to neuromorphic computing. ${ }^{7}$ These applications exploit the fast and reversible transformation between the amorphous and crystalline phases upon heating. In the electronic non-volatile memory (phase change memories, PCMs) the two states of the memory can be discriminated, thanks to a large change in the electrical resistivity across the phase change. Although $\mathrm{Ge}_{2} \mathrm{Sb}_{2} \mathrm{Te}_{5}$ (GST) is presently the material of choice for electronic applications, the search of new alloys with better functional properties is a very active area of research. To make PCMs suitable for applications in automotive technologies, for instance, the stability of the amorphous phase against recrystallization must be extended above $125^{\circ} \mathrm{C}$, but still preserving a fast phase switching. ${ }^{5}$

The InSbTe (IST) ternary system is currently investigated as a phase change material because of the presence of compositions with high melting ${ }^{8}$ and crystallization temperatures. ${ }^{9}$ The $\mathrm{In}_{3} \mathrm{SbTe}_{2}$ composition along the pseudobinary InSb-InTe tie line, in particular, was shown to undergo a sequence of transformations from the amorphous to the crystal through several intermediate phases with different resistivities ${ }^{10-12}$ that might be exploited for multibits PCMs. ${ }^{13,14}$

\footnotetext{
a) Present address: University of Zurich, Department of Chemistry, Winterthurerstrasse 190, CH-8057 Zurig, Switzerland.

b) Radboud University, Institute for Molecules and Materials, Heyendaalseweg 135, 6525AJ Nijmegen, The Netherlands.

c) Dynamics of Condensed Matter, University of Paderborn, Warburger Strasse 100, D-33098 Paderborn, Germany.

d) marco.bernasconi@mater.unimib.it
}

In a previous work ${ }^{15}$ we studied the structural properties of the amorphous phase of the $\mathrm{In}_{3} \mathrm{SbTe}_{2}$ compound by means of molecular dynamics simulation based on Density Functional Theory (DFT). ${ }^{16}$ The resulting amorphous phase featured a competition between octahedral-like bonding geometries typical of the rocksalt crystalline phase of $\mathrm{In}_{3} \mathrm{SbTe}_{2}$ and tetrahedral-like geometries similar to those present in the crystalline phase of the two binary compounds InTe and InSb, which is what the ternary system can be thought to be made of. ${ }^{15}$ These different bonding configurations are closely competing ${ }^{15}$ as occurs for the octahedral- and tetrahedral-like configurations of Ge atoms in DFT models of amorphous GeSbTe alloys ${ }^{17-19}$ and of In in amorphous $\mathrm{InGeTe}_{2} \cdot{ }^{20}$ In all the previous DFT studies mentioned above, some variants of the most popular Perdew-Burke-Ernzerhof $(\mathrm{PBE})^{21}$ exchange and correlation functional were used. It has been argued, however, whether different choices of the exchange-correlation (xc) functional might sizably change the tetrahedra to octahedra ratio. For instance, the addition of the semi-empirical van der Waals correction according to Grimme ${ }^{22}$ was shown to provide a better agreement with experiments on the structure of liquid and amorphous $\mathrm{Ge}_{15} \mathrm{Te}_{85}$ which feature long and weakly interacting $\mathrm{Te}-\mathrm{Te}$ chains. ${ }^{23}$ Although this semi-empirical van der Waals correction has a marginal effect on the structure of the alloy with the GeTe composition in which $\mathrm{Te}-\mathrm{Te}$ chains are absent, the more complex van der Waals density functional proposed by Lee et al. $^{24}$ (denoted as vdW-DF2) was shown to better reproduce the bond length of GeTe as well, giving rise to a more defined first coordination shell ${ }^{25}$ with respect to the models generated with PBE or other semi-local functionals. Still, the vdW-DF2 functional does not change sizably the ratio between tetrahedra and octahedra in amorphous GeTe. ${ }^{25}$ On the other hand the 
Becke-Lee-Yang-Parr (BLYP) ${ }^{26}$ functional has been shown to better reproduce the structural properties of liquid and amorphous $\mathrm{GeSe}_{2}$ and $\mathrm{GeSe}$ compounds with respect to the PBE functional. ${ }^{27,28}$ In particular, the stronger electron localization of the BLYP functional leads to models of liquid GeSe with a larger fraction of tetrahedra with respect to models generated with the PBE functional. ${ }^{28}$ These results on selenides raise the question on whether a similar difference in the tetrahedra to octahedra ratio between PBE and BLYP models might arise for phase change tellurides as well. Concerning GeSbTe alloys, it was shown that the PBE and BLYP functionals yield very similar structural properties for amorphous $\mathrm{Ge}_{2} \mathrm{Sb}_{2} \mathrm{Te}_{5},{ }^{29}$ although previous simulations with smaller simulation cells ${ }^{30}$ suggested a slightly larger concentration of tetrahedra with the use of the BLYP functional.

In this paper, we investigate the influence of the exchange and correlation functional on the structure of amorphous $\mathrm{In}_{3} \mathrm{SbTe}_{2}$ by comparing models generated by quenching from the melt with the BLYP functional with those generated with the PBE functional and discussed in our previous work. ${ }^{15}$ For the sake of completeness we have also revisited with the use of the BLYP functional the structural properties of the amorphous phase of the binary InSb compound that we have simulated with the PBE functional in our previous works. ${ }^{31,32}$ As opposed to what occurs in GeSbTe alloys, it turns out that in the case of InSb the use of the BLYP functional brings the theoretical results in better agreement with experiment by favoring the tetrahedral-like environment with respect to the octahedral-like ones. A similarly larger fraction of tetrahedral configurations is also found in the BLYP models of the ternary InSbTe alloys with respect to those generated with the PBE functional.

\section{COMPUTATIONAL METHODS}

The amorphous models were generated by quenching from the melt within DFT-based molecular dynamics simulations by using the scheme of Kühne et $a .^{33}$ In the spirit of the Car-Parrinello (CP) ${ }^{34}$ approach the wavefunctions are not self-consistently optimized during the dynamics. However, in contrast to CP, large integration time steps can be used in the simulation. This scheme leads to a slightly dissipative dynamics of the type $-\gamma_{D} \dot{\mathbf{R}}_{I}$, where $\mathbf{R}_{I}$ are the ionic coordinates. In Refs. 33 and 35 it is shown how to compensate for this dissipation by a modified Langevin equation in order to obtain a correct canonical sampling. This scheme is implemented in the $\mathrm{CP} 2 \mathrm{~K}$ suite of programs. ${ }^{36,37}$ We used the BLYP exchange-correlation functional and Goedecker-type pseudopotentials ${ }^{38}$ with three, five, and six valence electrons for $\mathrm{In}, \mathrm{Sb}$, and Te, respectively. The Kohn-Sham (KS) orbitals were expanded in a Triple-ZetaValence plus Polarization (TZVP) Gaussian-type basis set, while the charge density has been expanded in a planewave basis set with a cut-off of $100 \mathrm{Ry}$ to efficiently solve the Poisson equation within periodic boundary conditions using the Quickstep scheme. ${ }^{36,37}$ Brillouin zone integration was restricted to the supercell $\Gamma$-point. An integration time step of $2 \mathrm{fs}$ was used. Temperature was enforced by the Langevin thermostat mentioned above.
The InSb compound was modeled by a cubic supercell with 216 atoms at the density of the crystalline phase of 0.0295 atoms $/ \AA^{3}\left(5.775 \mathrm{~g} / \mathrm{cm}^{3}\right)$ as in previous simulations with the PBE functional ${ }^{31}$ and in molecular dynamics simulations with a classical interatomic potential. ${ }^{39}$ The liquid model was first equilibrated at $1000 \mathrm{~K}$ and then quenched to $300 \mathrm{~K}$ in $100 \mathrm{ps}$. We remark that the equilibrium lattice parameter and bulk modulus of crystalline InSb in the zincblend geometry are $6.575 \AA$ and $35 \mathrm{GPa}$ with the BLYP functional and $6.622 \AA$ and $35 \mathrm{GPa}$ with the PBE functional, to be compared with the experimental values of $6.479 \AA$ and $46.5 \mathrm{GPa}^{40}$

In our previous work, ${ }^{15}$ we generated models of a$\mathrm{In}_{3} \mathrm{SbTe}_{2}$ by quenching from the melt at two fixed densities with the PBE functional. In the lack of experimental information on the density we initially fixed the density of the liquid to the value $6.448 \mathrm{~g} / \mathrm{cm}^{3}\left(0.0323\right.$ atoms $\left./ \AA^{3}\right)$ which is about $7 \%$ lower than the density of $6.96 \mathrm{~g} / \mathrm{cm}^{3}$ that can be assigned to the ideal rocksalt crystal with the experimental lattice parameter of 6.126(1) $\AA^{41,42}$ A similar increase in density upon crystallization of the amorphous is found in phase change GeSbTe alloys. ${ }^{2}$ An amorphous model was then generated by quenching the liquid at fixed density from $1000 \mathrm{~K}$ to $300 \mathrm{~K}$ in about $250 \mathrm{ps}$. The theoretical equilibrium density of the amorphous model was then obtained from a Murnaghan fitting of the energy-volume data of the optimized geometries at zero temperature. The resulting theoretical equilibrium density is $5.75 \mathrm{~g} / \mathrm{cm}^{3}\left(0.0288\right.$ atoms $\left./ \AA^{3}\right)$ which is about $11 \%$ lower than our starting density of the liquid. A second amorphous model was then generated by quenching from the melt in about $350 \mathrm{ps}$ at the fixed density of $5.75 \mathrm{~g} / \mathrm{cm}^{3}$.

To investigate the effect of a different choice of the $x c$ functional, in this work the $\mathrm{In}_{3} \mathrm{SbTe}_{2}$ compound was modeled by a cubic supercell with 300 atoms at the same density of $5.75 \mathrm{~g} / \mathrm{cm}^{3}\left(0.0288\right.$ atoms $\left./ \AA^{3}\right)$ or $6.448 \mathrm{~g} / \mathrm{cm}^{3}$ $\left(0.0323\right.$ atoms $\left./ \AA^{3}\right)$ of the models generated previously with the PBE functional. ${ }^{15}$ The liquid was first equilibrated at $1000 \mathrm{~K}$ and then an amorphous model was obtained by quenching to $300 \mathrm{~K}$ in $100 \mathrm{ps}$.

We also performed constant pressure simulations during the quenching by using the Inverse Simulated Annealing (ISA) method introduced recently. ${ }^{32,43}$ The ISA method is a Monte Carlo (MC) annealing scheme, designed to combine the accuracy of $a b$ initio calculations with the reverse MC technique $^{44,45}$ in order to efficiently generate low energy structures in best agreement with available experimental data. Here we will use ISA merely as a minimization tool, i.e., without any experimental constraints. As such, ISA has been shown to be quite competitive in the application to amorphous phases with respect the more standard annealing procedure using molecular dynamics in the sense that it yields very similar structures but in significantly less computation time. ${ }^{43}$ The hybrid MC-based ISA method introduced in our previous works ${ }^{32,43}$ is a "fuzzy" hybrid MC method with all atom trial moves generated by a single, energy conserving, velocity-Verlet MD step but with a randomly chosen time step $d t \in\left(0, d t_{\max }\right)$, where $d t_{\max }$ is adjusted on-the-fly to achieve an acceptance of 50\%. To perform isobaric simulations, also volume moves are allowed with an acceptance probability 
equal to that in standard MC simulation within the NPT ensemble. Typically, $d t_{\max }$ turns out to be much larger (up to an order of magnitude) than in a normal MD simulation. Further details can be found in Ref. 32 .

In constant pressure simulations as well as in constant volume simulations of $\mathrm{In}_{3} \mathrm{SbTe}_{2}$, we also added van der Waals (vdW) corrections according to Grimme ${ }^{22}$ to better reproduce the equilibrium density. The constant pressure ISA simulations provided a model of a- $\mathrm{In}_{3} \mathrm{SbT}_{2}$ at zero pressure at an equilibrium density of $5.554 \mathrm{~g} / \mathrm{cm}^{3}\left(0.0278\right.$ atoms $\left./ \AA^{3}\right)$. The structural properties of the BLYP models at the two densities of $5.75 \mathrm{~g} / \mathrm{cm}^{3}$ and $5.554 \mathrm{~g} / \mathrm{cm}^{3}$ are, however, very similar as discussed in Sec. III B.

\section{RESULTS}

\section{A. InSb}

In our previous work ${ }^{31}$ we generated models of a-InSb by quenching from the liquid phase within DFT-MD simulation using the PBE functional.

It was found that short quenches $(50 \mathrm{ps}-130 \mathrm{ps}$ from the melting point to $300 \mathrm{~K}$ ) with an accurate double-zeta-valence plus polarization (DZVP) basis set give rise to a large fraction of octahedral-like local geometries and pair correlation functions (PCFs) in strong disagreement with experiments. Instead, a quench 350 ps long with a more contracted basis set leads to a model lower in energy (once optimized with the accurate DZVP basis set), with mostly tetrahedral local geometries and in good agreement with the experimental pair correlation function inferred from x-ray data. ${ }^{46}$ However, it later turned out that the mostly tetrahedral model of Ref. 32 was actually resulting from the use of the too contracted basis set rather than from the long quenching time of $350 \mathrm{ps}{ }^{32}$ In fact, simulations with similarly long quenches and the accurate DZVP basis set invariably lead to models with a large fraction of octahedrallike local geometries, in one case also lower in energy than the mostly tetrahedral-like model. We remark that the accurate DVZP and the TZVP basis sets used here have been extensively validated against calculations with a plane wave basis sets and the same pseudopotentials which actually revealed that the more contracted basis set used only in the quenching protocol employed in Ref. 31 was not sufficiently accurate.

The octahedral-like geometries have bonding angles at about $90^{\circ}$ and $180^{\circ}$ but coordination lower than six, actually four or five. These structures have been referred to as defective octahedra in previous DFT works on InSbTe alloys ${ }^{15}$ and other amorphous tellurides. ${ }^{17-20,47-54}$ Three-fold coordination with bonding angles of $90^{\circ}$ corresponds instead to a pyramidal geometry which is also found for Sb. Snapshots of tetrahedral, defective-octahedral and pyramidal geometries are shown in Fig. 1. Actually, it is known experimentally that aInSb crystallizes in a cubic rocksalt phase under moderate pressure of 1-1.3 $\mathrm{GPa}^{55}$ which would correspond to a density increase of only $4 \%$ by assuming a crystalline-like bulk modulus of $35 \mathrm{GPa} \cdot{ }^{31}$ It is therefore conceivable that a close competition arises between octahedral-like and tetrahedrallike local geometries. In Ref. 32 we reported on the energetics and structure of different models of a-InSb generated in

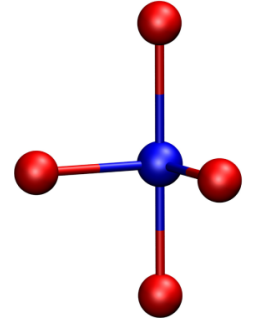

(a)

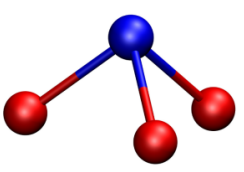

(b)

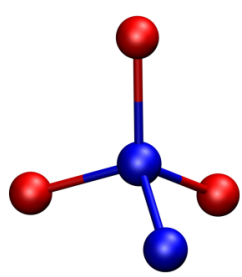

(c)
FIG. 1. Snapshot of Sb atoms in (a) defective octahedra, (b) pyramids, and (c) tetrahedra. $\mathrm{Sb}$ atoms are depicted by blue spheres, while In atoms by red spheres.

different manners with the PBE functional. The lowest energy model (MD-2) obtained with the accurate DZVP basis set and a long quench $(350 \mathrm{ps})$ features a mostly defective-octahedral local geometry. However, the model discussed in Ref. 31, and generated with the more contracted basis set, is mostly tetrahedral and in good agreement with experiments, although it is higher in energy than the model MD-2. ${ }^{32}$ It actually turns out that the model generated with the BLYP functional is also mostly tetrahedral and very similar to the PBE model reported in Ref. 31. The total pair correlation function of the a-InSb models generated with BLYP and PBE (lowest energy MD-2 model, cf., Ref. 32) at the same density of $5.775 \mathrm{~g} / \mathrm{cm}^{3}$ is compared with the experimental x-ray data from Ref. 46 in Fig. 2. The BLYP model much better reproduces the experimental data than the lowest energy PBE (MD-2) model generated with the accurate basis set.

Information on the differences in the structural properties between the two models (BLYP and PBE MD-2) is gained from the comparison of the partial PCFs (Fig. 3), the average partial coordination numbers (Table I), the fraction of the different types of bonds (Table I), the distribution of the coordination numbers (Fig. 4), and the bond angle distribution function (Fig. 5). The partial PCFs of the BLYP model display a more compact first coordination shell with well defined minima that allow an unambiguous definition of the bonding cutoff for the calculation of the coordination numbers. On the contrary, the coordination shells of the PBE model look very

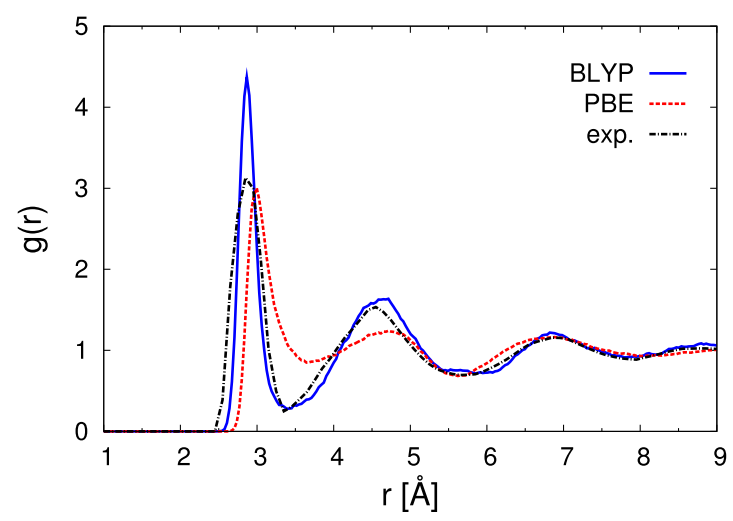

FIG. 2. Total pair correlation function of $\mathrm{a}-\mathrm{InSb}$ at the density of $5.775 \mathrm{~g} / \mathrm{cm}^{3}$ computed with the BLYP (continuous line) and the PBE functional (dashed line) compared with the experimental x-ray data from Ref. 46 (dotted-dashed line). 


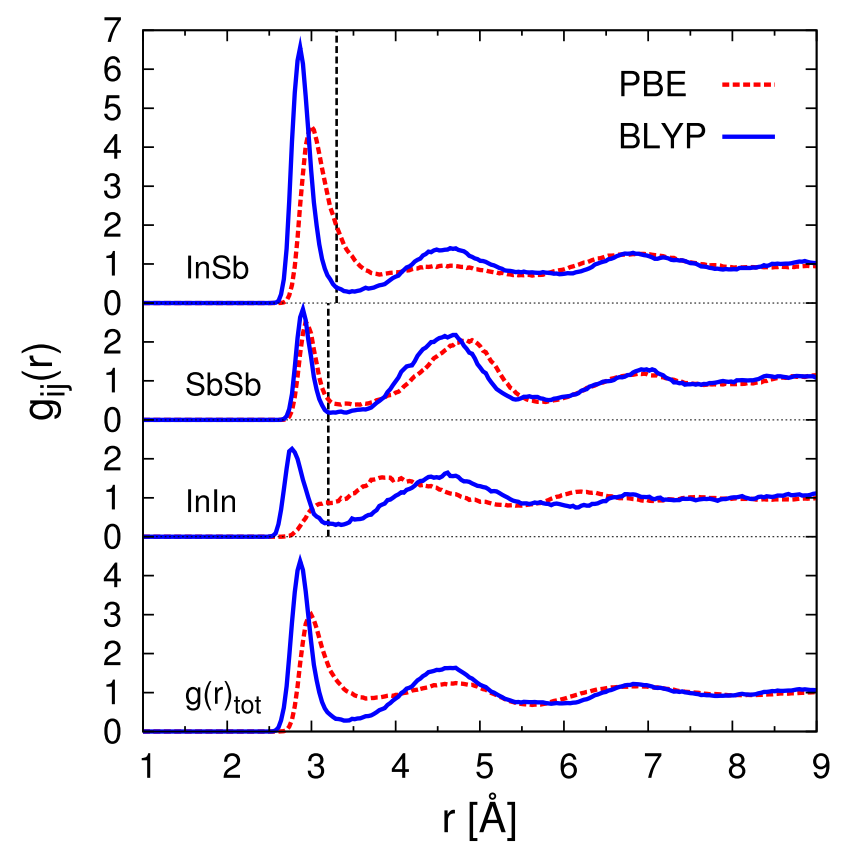

FIG. 3. Total and partial pair correlation functions of a-InSb at the density of $5.775 \mathrm{~g} / \mathrm{cm}^{3}$ computed with the BLYP functional (continuous line) and the PBE functional (dashed line). The vertical lines are the bonding cutoff used to define the coordination numbers, namely, $3.20 \AA$, $3.30 \AA$, and $3.20 \AA$, for the In-In, In-Sb, and Sb-Sb bonds, respectively, in the BLYP model and (not shown) $3.30 \AA$, $3.50 \AA$, and $3.25 \AA$, for the In-In, In-Sb, and Sb-Sb bonds, respectively in the PBE model.

broad with a first minimum at an anomalously high distance which cannot correspond to really bonded atoms. In the PBE model the choice of the bonding cutoff cannot be reliably assigned on the basis of the bonding geometry only, but it would require the analysis of the electronic structure as well. We have not performed such an analysis here because it does not seem to be so critical in the case of the BLYP models we are interested in. The choice of the bonding cutoff for the PBE model is therefore subject to an arbitrariness which, however, does not affect the overall conclusions drawn below on the comparison between the PBE and BLYP results.

The coordination numbers are larger in the PBE model than in the BLYP models, in particular due to the presence of several 5-fold coordinated $\mathrm{Sb}$ atoms (cf., Fig. 4). The distribution of bond angles for $\mathrm{Sb}$ is also strikingly different in the BLYP and PBE models due to a different ratio of tetrahedral and defective octahedral configurations. Apparently the PBE functional strongly favors the defective

TABLE I. Average coordination numbers for different pairs of atoms computed from the partial pair correlation functions (cf., Fig. 3) of the BLYP model of a-InSb at $5.775 \mathrm{~g} / \mathrm{cm}^{3}$. The percentage of the different types of bonds with respect to the total number of bonds is also given. Data for the model generated with the PBE functional at the same density are reported in parenthesis.

\begin{tabular}{|c|c|c|c|c|c|c|}
\hline \multicolumn{4}{|c|}{ Average coordination numbers } & \multicolumn{3}{|c|}{ Types of bonds (\%) } \\
\hline & With In & With Sb & Total & & With In & With Sb \\
\hline In & $1.0(0.6)$ & $2.9(3.4)$ & $3.9(4.0)$ & In & $13(7)$ & $74(80)$ \\
\hline $\mathrm{Sb}$ & $2.9(3.4)$ & $1.0(1.1)$ & $3.9(4.5)$ & $\mathrm{Sb}$ & & $13(13)$ \\
\hline
\end{tabular}

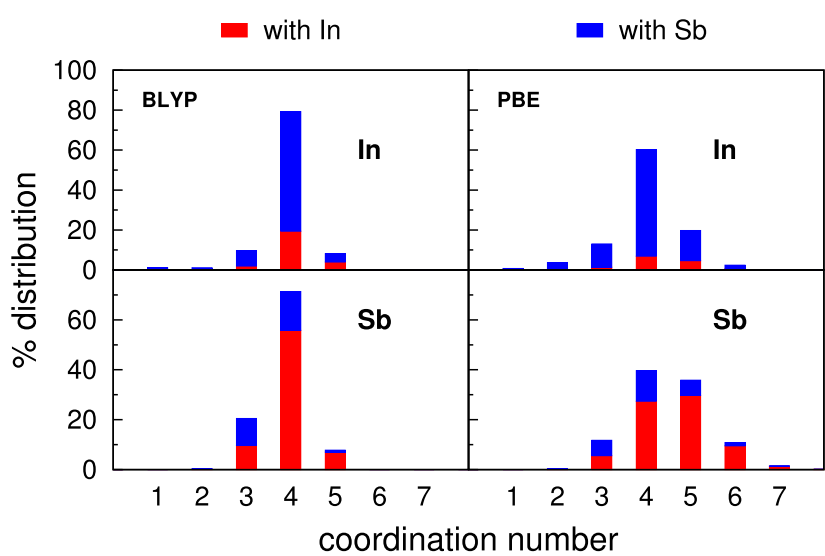

FIG. 4. Distribution of the coordination numbers of a-InSb at the density of $5.775 \mathrm{~g} / \mathrm{cm}^{3}$ computed with the BLYP functional (left panel) and the PBE functional (right panel). The contributions from the different pairs of atoms are indicated by different colors.

octahedral configuration for $\mathrm{Sb}$ which also leads to a broader pair correlation function and a less defined first coordination shell.

To quantify the fraction of defective-octahedral and tetrahedral configurations we used the local order parameter $q$ introduced in Ref. 56 as an indicator of the tetrahedricity of the bonding geometry. It is defined by $q=1-\frac{3}{8} \sum_{i>k}\left(\frac{1}{3}\right.$ $\left.+\cos \theta_{i j k}\right)^{2}$ where the sum runs over the pairs of atoms bonded to a central atom $j$ and forming a bonding angle $\theta_{i j k}$. The order parameter evaluates to $q=1$ for the ideal tetrahedral geometry, to $q=0$ for the six-coordinated octahedral site, and to $q=5 / 8$ for a four-coordinated defective octahedral site. The distribution of the $q$ parameter for the BLYP and PBE models is compared in Fig. 6. The concentration of atoms in tetrahedral geometry can be obtained by integrating the $q$ distribution for 4-coordinated atoms from $q=0.8$ to its upper limit as discussed in Refs. 15, 20, and 52, 53. We obtain a fraction of In and $\mathrm{Sb}$ atoms in tetrahedral geometry of $67 \%$ and $48 \%$ for the BLYP model and of $43 \%$ and $11 \%$ for the PBE (MD-2) model. The BLYP model is therefore mostly tetrahedral while the PBE model mostly features defective octahedral environments.

Concerning the medium range order, we report the distribution of ring length in Fig. 7 computed according to Ref. 57. In the PBE model the distribution of rings is rather broad, with a large abundance of 4-, 5-, and 6-membered rings. The 4-membered ring is the building block of the cubic rocksalt phase and corresponds to $\sigma_{p}$-bonds in defective

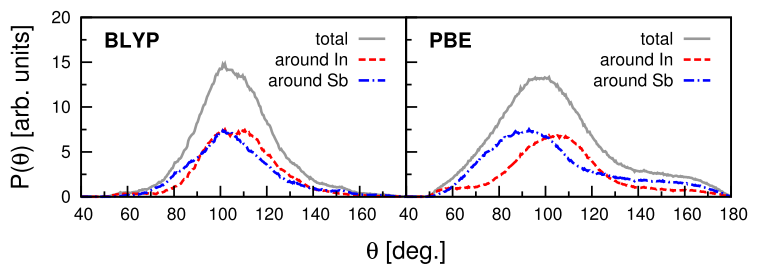

FIG. 5. Bond angles distribution of a-InSb at the density of $5.775 \mathrm{~g} / \mathrm{cm}^{3}$ computed with the BLYP (left panel) and the PBE functional (right panel). The total distribution (continuous line) is resolved into the contributions from the bonds made by In (dashed line) and Sb (dotted-dashed line) atoms. 


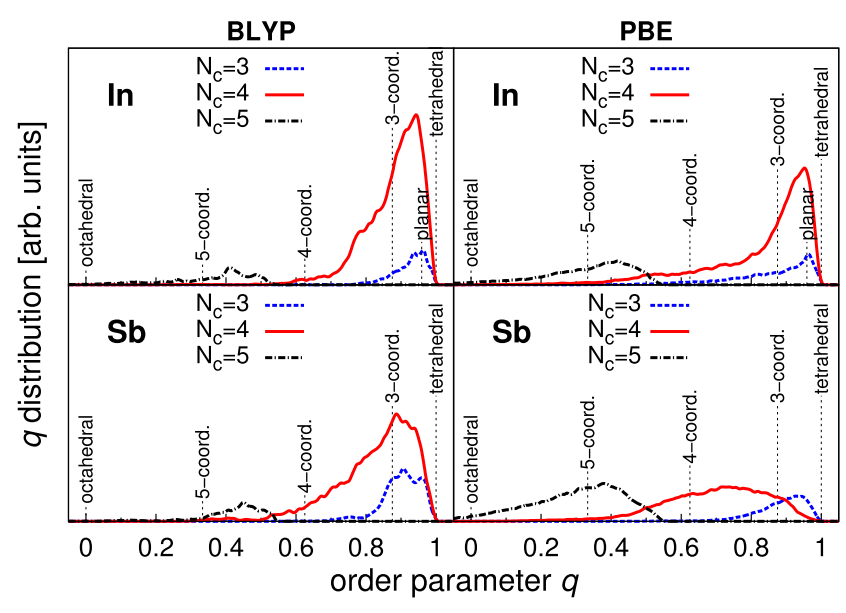

FIG. 6. Distribution of the local order parameter $q$ for tetrahedricity (see text) for a-InSb at the density of $5.775 \mathrm{~g} / \mathrm{cm}^{3}$ computed with the BLYP (left panel) and the PBE functional (right panel). The distribution for In and Sb atoms are given separately in the upper and lower panels and are further resolved in the contributions from atoms with different coordination $\left(\mathrm{N}_{c}\right)$. Vertical dashed lines indicate the values of $q$ for selected ideal defectiveoctahedral geometries and for the tetrahedral geometry. By integrating the $q$ distribution from 0.8 to 1.0 we obtain a fraction of In and $\mathrm{Sb}$ atoms in tetrahedral geometry of $67 \%$ and $48 \%$ for the BLYP model and of $43 \%$ and $11 \%$ for the PBE model.

octahedra. It is the most abundant ring in GST and GeTe. ${ }^{17-19,50}$ In the BLYP model the most abundant rings are the five- and six-membered ones, typical of a mostly tetrahedral network.

Homopolar $\mathrm{Sb}-\mathrm{Sb}$ bonds are arranged in clusters or chains whose distribution is reported in Fig. S1 in the supplementary material (SM). ${ }^{64}$ A large 13-atom cluster (a branched chain), present in the model generated with the BLYP functional, is shown in Fig. S2 in the SM.

In summary, the model of a-InSb generated with the BLYP functional has a mostly tetrahedral network and reproduces well the experimental PCF. The tetrahedral bonding geometry is favored with respect to the octahedral-like geometry by the BLYP functional. In fact, once relaxed with the BLYP functional, the mostly octahedral MD-2 model generated with the PBE functional is higher in energy by about $41 \mathrm{meV} /$ atom with respect to the tetrahedral-like model generated with the BLYP one. The reverse is true if we optimize with the PBE functional the tetrahedral model which becomes $29 \mathrm{meV} /$ atom higher in energy than the MD-2 model at the PBE level.

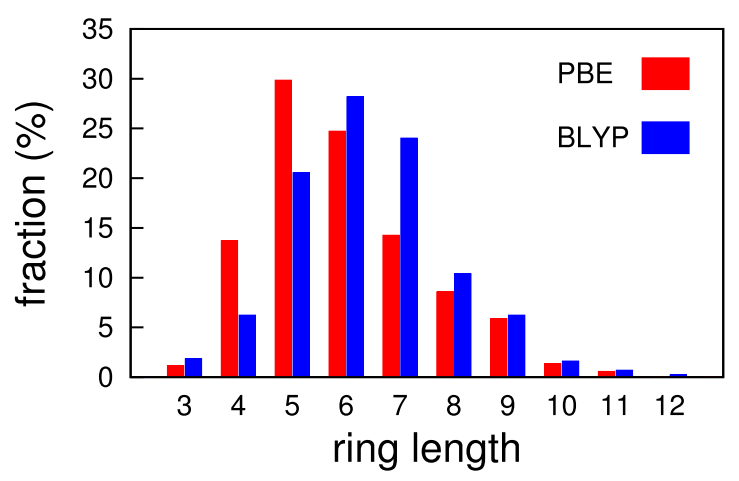

FIG. 7. Ring distribution function of a-InSb at the density of $5.775 \mathrm{~g} / \mathrm{cm}^{3}$ computed with the BLYP and PBE functionals.
The effect of vdW interactions on the structural properties was also investigated by generating a model of a-InSb at the same density of $5.775 \mathrm{~g} / \mathrm{cm}^{3}$ by quenching from the melt in 100 ps with BLYP simulations and by including vdW interactions according to Grimme. ${ }^{22}$ The only difference with respect to the BLYP model without vdW correction is a fraction of tetrahedra about $2 \%$ lower for In atoms and $2 \%$ higher for $\mathrm{Sb}$ atoms which are, however, within the uncertainties due to the small simulation cell.

We remark that these two models are generated at the same fixed density. The vdW corrections according to Grimme have thus a negligible effect on the short range structure at fixed volume, but they do affect the equilibrium volume. The theoretical equilibrium density of the zincblend crystal increases by about $6 \%$ with respect to BLYP calculations without vdW correction. To investigate the effect of the density on the structure of a-InSb we generated another amorphous model at the fixed density of $5.355 \mathrm{~g} / \mathrm{cm}^{3}$ which differs by about $8 \%$ with respect to the $5.775 \mathrm{~g} / \mathrm{cm}^{3}$ density of the models discussed above which in turn is slightly higher than the experimental density of $5.66 \mathrm{~g} / \mathrm{cm}^{3}$ of the as-deposited samples. ${ }^{46}$ The partial coordination numbers of this second model are equal to those given in Table I within the figures given there. Therefore we can conclude that vdW corrections have an overall negligible effect on the short range topology of a-InSb.

These new results on InSb point to a deficiency of the PBE exchange-correlation functional when dealing with the close competition in energy between tetrahedral-like and octahedrallike configurations, which is probably also responsible for the tetrahedral-to-octahedral transition observed experimentally in a-InSb under moderate pressure. ${ }^{55}$

\section{B. $\ln _{3} \mathrm{SbTe}_{2}$}

The crystalline cubic phase of $\mathrm{In}_{3} \mathrm{SbTe}_{2}$ is thermodynamically stable only in the temperature range $470{ }^{\circ} \mathrm{C}-570{ }^{\circ} \mathrm{C}$, but it can be recovered upon quenching at normal conditions, bypassing the decomposition into InTe and InSb expected below $470{ }^{\circ} \mathrm{C}$. The crystal structure of cubic $\mathrm{In}_{3} \mathrm{SbTe}_{2}$ was assigned to the $\mathrm{Fm} \overline{3} \mathrm{~m}$ space group with a lattice constant of $6.126(1) \AA$ and a rocksalt geometry ${ }^{41}$ with In occupying the cation sublattice and $\mathrm{Sb}$ and $\mathrm{Te}$ atoms occupying the anion sublattice in a random manner as shown by recent $\mathrm{x}$-ray and neutron diffraction experiments. ${ }^{42}$ The crystal is metallic with a conductivity of $3.2 \cdot 10^{4} \mathrm{~S} / \mathrm{cm}$ at normal conditions. ${ }^{42}$

In our previous work $^{15}$ we reported a theoretical equilibrium density about $10 \%$ lower than in experiments as computed from the equation of state of the cubic crystal in a 216-atom supercell with the PBE functional. In this calculation the Brillouin zone integration was restricted to the $\Gamma$-point only which, however, turns out to be insufficient given the metallic nature of the compound. In fact, the theoretical cubic lattice parameter turns out to be $6.05 \AA$, in good agreement with experiments, once the system is modeled in a hexagonal cell with six planes stacked along the $c$ axis as In-Sb-In-Te-In-Te- and six atoms per unit cell and the Brillouin zone is integrated over a $8 \times 8 \times 4$ uniform mesh ${ }^{58}$ by using the Quantum-espresso suite of programs. ${ }^{59}$ The 
hexagonal cell was optimized by fixing the c/a ratio to the value $(2 \sqrt{6})$ expected for a cubic-like symmetry. A similar hexagonal setup was used in a recent DFT work on the chemical bonding in $\mathrm{In}_{3} \mathrm{SbTe}_{2} .{ }^{60}$

The octahedral-like bonding geometry of the cubic $\mathrm{In}_{3} \mathrm{SbTe}_{2}$ crystal has to be contrasted with the tetrahedral bonding geometry of the binary compounds $\operatorname{InTe}^{61}$ and InSb. In fact, InSb crystallizes in a zinc-blende structure, while crystalline InTe is made of chains of edge-sharing $\mathrm{InTe}_{4}$ tetrahedra intercalated by weakly bound, interstitial-like In ions. ${ }^{61}$

In Ref. 15 we have shown that the models of the amorphous phase of $\mathrm{In}_{3} \mathrm{SbTe}_{2}$ generated with the PBE functional feature both the octahedral (defective) local geometry similar to those present in the ternary cubic crystal and the tetrahedral geometries present in the two parent binary compounds. The abundance of the different bonding geometries in the amorphous phase was, however, strongly dependent on the density in the range $6.448-5.75 \mathrm{~g} / \mathrm{cm}^{3}$ that we investigated. At high density the bonding topology was mostly octahedral-like (18\% of tetrahedral In atoms) while at low density a fraction as large as $41 \%$ of In atoms had a tetrahedral-like bonding geometry.

The models of a- $\mathrm{In}_{3} \mathrm{SbTe}_{2}$ generated here with the BLYP functional at the same two densities of $6.448 \mathrm{~g} / \mathrm{cm}^{3}$ $\left(0.0323\right.$ atoms $\left./ \AA^{3}\right)$ and $5.75 \mathrm{~g} / \mathrm{cm}^{3}\left(0.0288\right.$ atoms $\left./ \AA^{3}\right)$ investigated previously (cf., Sec. II) show a much more pronounced tetrahedral character with respect to the PBE models. In fact, $63 \%$ and $70 \%$ of In atoms are in a tetrahedral geometry at high and low densities. A similar fraction of $66 \%$ of In atoms are in a tetrahedral geometry at the equilibrium density of $5.554 \mathrm{~g} / \mathrm{cm}^{3}\left(0.0278\right.$ atoms $\left./ \AA^{3}\right)$ obtained from the constant pressure ISA simulations. The dependence of the structural properties on the density is thus much less striking with the BLYP functional than with the PBE functional.

Information on the differences in the structural properties between the models generated with the two functionals at the

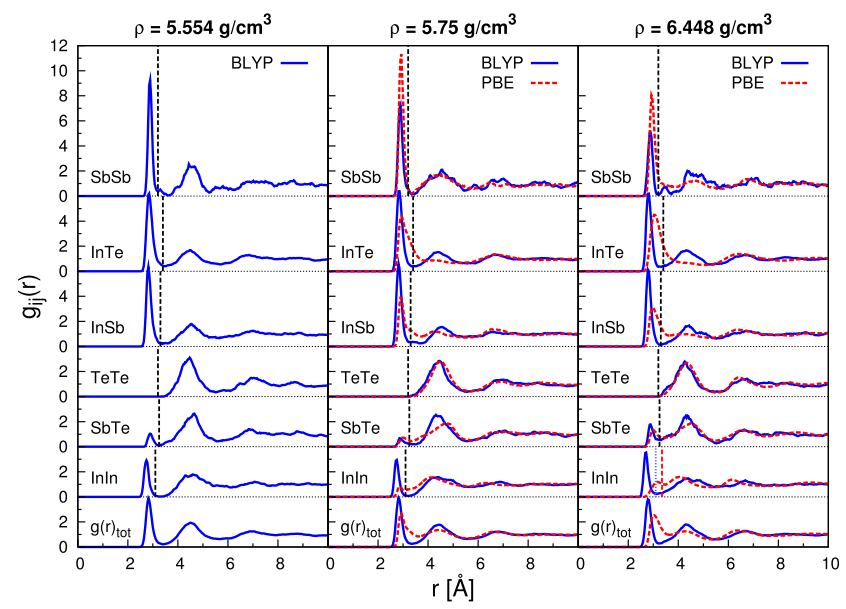

FIG. 8. Total and partial pair correlation functions of $\mathrm{a}-\mathrm{In}_{3} \mathrm{SbTe}_{2}$ models generated with the BLYP (continuous lines) and PBE (dashed lines) functionals at the density of $5.554 \mathrm{~g} / \mathrm{cm}^{3}$ (left panel), $5.75 \mathrm{~g} / \mathrm{cm}^{3}$ (central panel), or $6.448 \mathrm{~g} / \mathrm{cm}^{3}$ (right panel). The vertical lines are the bonding cutoff used to define the coordination numbers $(3.10$ or $3.35 \AA$, $3.30 \AA, 3.40 \AA, 3.25 \AA$, $3.20 \AA$, and $3.20 \AA$, for the In-In, In-Sb, In-Te, Sb-Te, Sb-Sb, and Te-Te bonds, respectively).
TABLE II. Average coordination numbers for different pairs of atoms computed from the partial pair correlation functions (cf., Fig. 8) for a- $\mathrm{In}_{3} \mathrm{SbTe}_{2}$ models generated with the BLYP functional and the PBE functional (in parenthesis) at the density of $5.75 \mathrm{~g} / \mathrm{cm}^{3}$ (upper table), $6.448 \mathrm{~g} / \mathrm{cm}^{3}$ (central table), and $5.554 \mathrm{~g} / \mathrm{cm}^{3}$ (lower table).

\begin{tabular}{lcccc}
\hline & With In & With Sb & With Te & Total \\
\hline $\mathrm{In}$ & $0.9(0.4)$ & $0.9(0.7)$ & $2.0(2.3)$ & $3.8(3.3)$ \\
$\mathrm{Sb}$ & $2.6(2.0)$ & $0.8(1.3)$ & $0.2(0.3)$ & $3.7(3.6)$ \\
$\mathrm{Te}$ & $3.0(3.4)$ & $0.1(0.2)$ & $0.0(0.0)$ & $3.1(3.6)$ \\
\hline & With In & With Sb & With Te & Total \\
\hline $\mathrm{In}$ & $1.3(1.0)$ & $0.9(0.8)$ & $2.0(2.7)$ & $4.1(4.5)$ \\
$\mathrm{Sb}$ & $2.6(2.4)$ & $0.7(1.2)$ & $0.6(0.6)$ & $3.9(4.2)$ \\
$\mathrm{Te}$ & $3.0(4.0)$ & $0.3(0.3)$ & $0.0(0.0)$ & $3.3(4.3)$ \\
\hline & With In & With Sb & With Te & Total \\
\hline $\mathrm{In}$ & 1.0 & 0.8 & 1.9 & 3.7 \\
$\mathrm{Sb}$ & 2.4 & 0.9 & 0.3 & 3.6 \\
$\mathrm{Te}$ & 2.9 & 0.1 & 0.0 & 3.0 \\
\hline \hline
\end{tabular}

two densities is gained from the comparison of the partial PCFs (Fig. 8), the average partial coordination numbers (Table II), the fraction of the different types of bonds (Table III), the distribution of the coordination numbers (Fig. 9), the bond angle distribution function (Fig. 10), and the distribution of the local order parameter $q$ for tetrahedricity (Fig. 11). The corresponding data for the BLYP model at the equilibrium density of $5.554 \mathrm{~g} / \mathrm{cm}^{3}$ obtained from constant pressure ISA simulations (cf., Sec. II) are also given for the sake of comparison.

The same caveats on the choice of the bonding cutoff for the PBE models discussed in Section III A for InSb hold here for the ternary alloy. In the BLYP models the first peak of the pair correlation function is higher and sharper, the coordination numbers are lower at all densities, and their distribution better peaked around four for $\mathrm{In} / \mathrm{Sb}$ and three for Te than in the PBE models. In the BLYP models there is also a slightly larger fraction of In-Sb and In-In bonds than in the

TABLE III. Percentage of the different types of bonds with respect to the total number of bonds for models of a- $\mathrm{In}_{3} \mathrm{SbTe}_{2}$ generated with the BLYP functional and the PBE functional (in parenthesis) at the density of $5.75 \mathrm{~g} / \mathrm{cm}^{3}$ (upper table), $6.448 \mathrm{~g} / \mathrm{cm}^{3}$ (central table), and $5.554 \mathrm{~g} / \mathrm{cm}^{3}$ (lower table).

\begin{tabular}{lccc}
\hline \hline & With In & With Sb & With Te \\
\hline $\mathrm{In}$ & $13(5)$ & $25(20)$ & $56(66)$ \\
$\mathrm{Sb}$ & & $4(6)$ & $2(3)$ \\
$\mathrm{Te}$ & & $0(0)$ \\
\hline & & With Sb & With Te \\
\hline $\mathrm{In}$ & With In & $23(18)$ & $52(62)$ \\
$\mathrm{Sb}$ & $17(12)$ & $3(4)$ & $6(4)$ \\
$\mathrm{Te}$ & & & $0(0)$ \\
\hline & & With Sb & With Te \\
\hline $\mathrm{In}$ & With In & 23 & 56 \\
$\mathrm{Sb}$ & 4 & & 3 \\
$\mathrm{Te}$ & & & 0 \\
\hline \hline
\end{tabular}




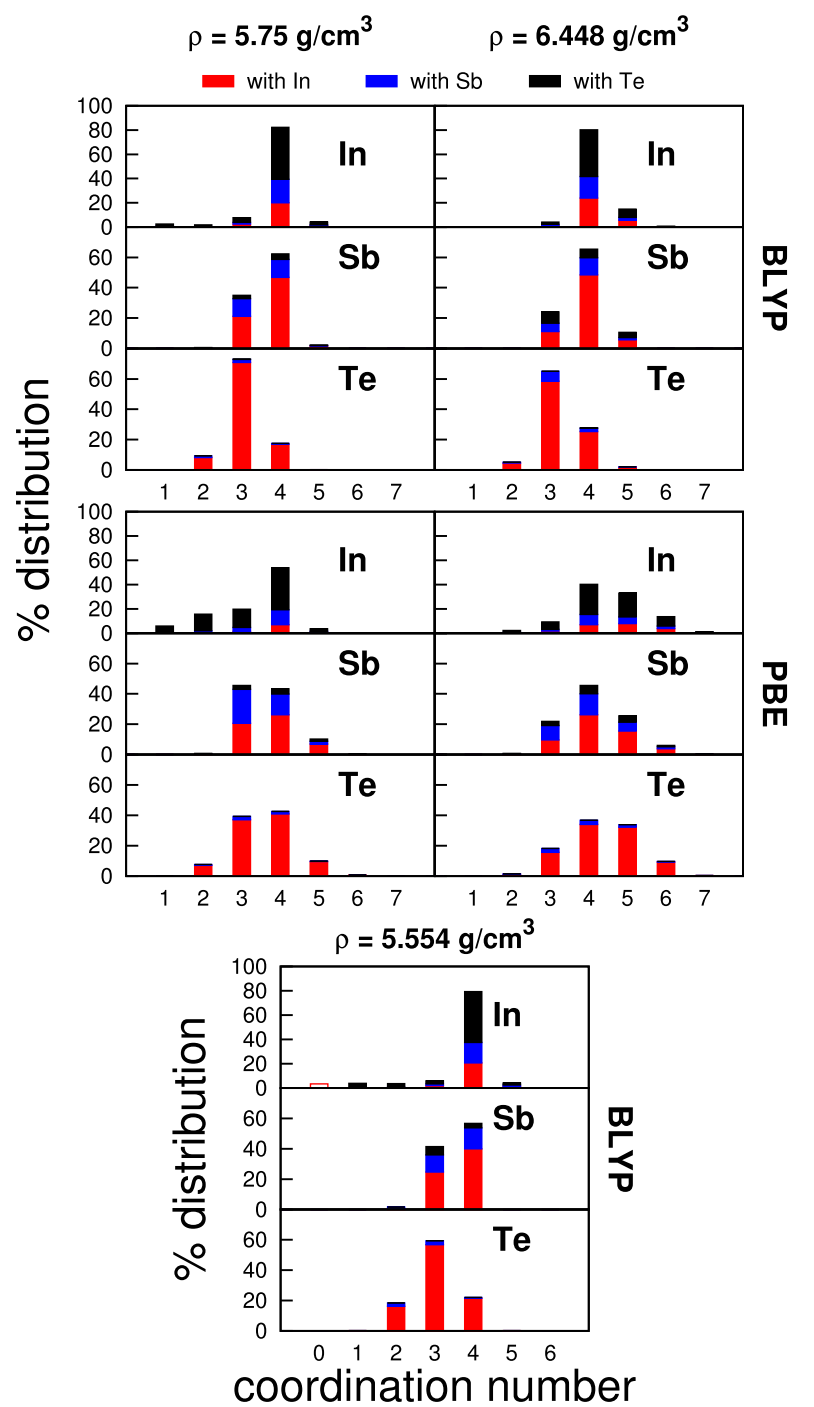

FIG. 9. Distribution of the coordination numbers of a- $\mathrm{In}_{3} \mathrm{SbTe}_{2}$ models generated with the BLYP (top panels) and PBE (central panels) functionals at the density of $5.75 \mathrm{~g} / \mathrm{cm}^{3}$ (left panels) or $6.448 \mathrm{~g} / \mathrm{cm}^{3}$ (right panels). The contributions from the different pairs of atoms are indicated by different colors. The data for the BLYP model at the theoretical equilibrium density of $5.554 \mathrm{~g} / \mathrm{cm}^{3}$ are reported in the bottom panel.

PBE models. The maximum of the bond angle distribution function is clearly shifted towards larger angles in the BLYP models due to a larger fraction of tetrahedra.

Integration of the $q$ order parameter for tetrahedricity leads to the estimates of the fraction of tetrahedral atoms as given at the beginning of the section and in the caption of Fig. 11. The distribution of the different types of tetrahedra (isolated, edge-, corner-sharing) is given in Table IV for the BLYP and PBE models. The overwhelming majority of tetrahedra is corner-sharing with a fraction of edge-sharing tetrahedra increasing with the overall fraction of tetrahedra, i.e., at lower density. The statistics of the different types of local environments are reported in Tables SI-SIII in the supplementary material for the BLYP models at the three densities. ${ }^{64}$ The distribution of ring length is shown in Fig. 12. The 4-membered ring typical of defective-octahedral structures is sizably lower in the mostly tetrahedral BLYP models than in the PBE ones.
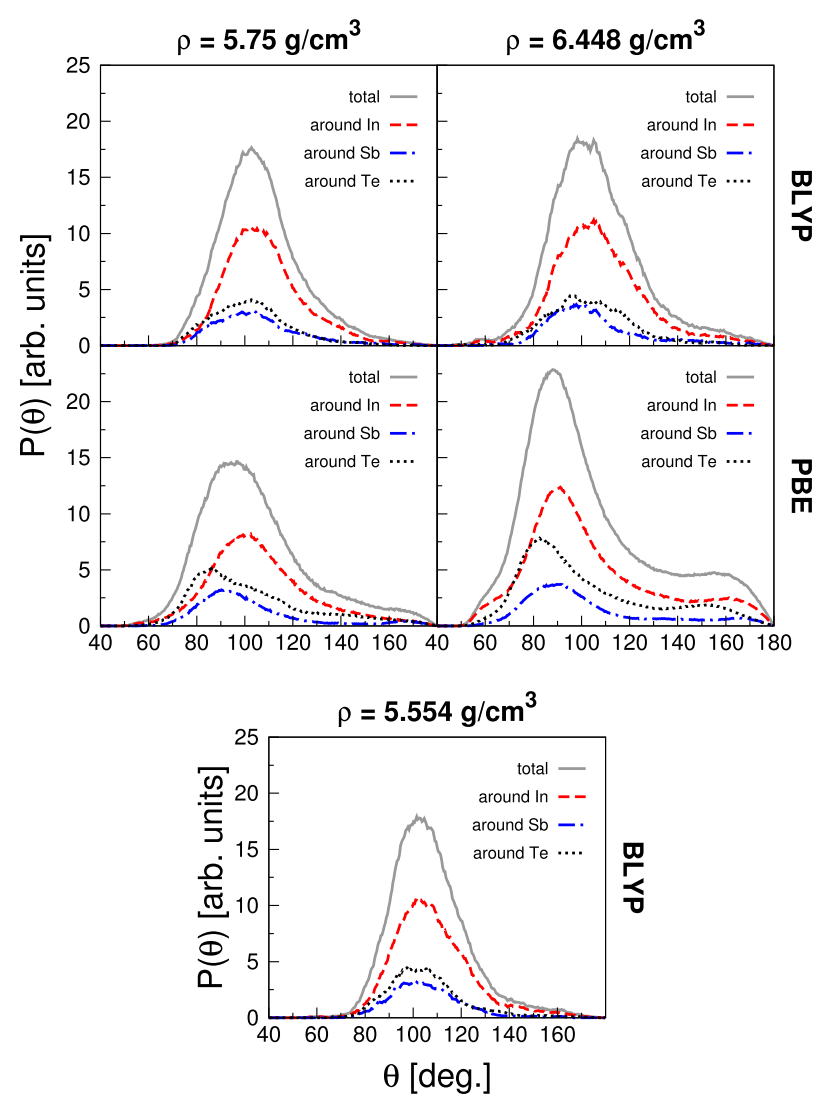

FIG. 10. Bond angles distribution of a- $\mathrm{In}_{3} \mathrm{SbTe}_{2}$ models generated with the BLYP functional (top panels) and the PBE functional (central panels) at the density of $5.75 \mathrm{~g} / \mathrm{cm}^{3}$ (left panels) or $6.448 \mathrm{~g} / \mathrm{cm}^{3}$ (right panels). The total distribution (continuous line) is resolved into the contributions from the bonds made by In (dashed line), Sb (dotted-dashed line), and Te (dotted line) atoms. The data for the BLYP model at the theoretical equilibrium density of $5.554 \mathrm{~g} / \mathrm{cm}^{3}$ are reported in the bottom panel.

The distribution of the size of Sb clusters in the models of a- $\mathrm{In}_{3} \mathrm{SbTe}_{2}$ is reported in Fig. S3 in the supplementary material. ${ }^{64}$ The number and size of $\mathrm{Sb}$ clusters are clearly much lower in a- $\mathrm{In}_{3} \mathrm{SbTe}_{2}$ than in a-InSb because of a lower fraction of $\mathrm{Sb}-\mathrm{Sb}$ bonds in the former system clearly related to an overall lower fraction of $\mathrm{Sb}$ atoms.

Overall, the BLYP models have a much larger fraction of tetrahedra than the PBE models which reflects in all changes in topology of the network and in a much milder dependence of the structure on the density than previously suggested on the basis of the PBE results. ${ }^{15}$

Electronic and vibrational properties: The electronic Density of States (DOS) of the BLYP models of a- $\mathrm{In}_{3} \mathrm{SbTe}_{2}$ is shown in Fig. 13 as computed from KS orbitals at the supercell $\Gamma$-point. The band gap decreases by increasing density and, as expected, it is overall smaller than the gap computed with the hybrid functional ${ }^{62}$ for the PBE models in Ref. 15.

The phonon DOS was computed from the diagonalization of the dynamical matrix obtained in turn from the variation of atomic forces due to finite atomic displacements $0.0053 \AA$ large. Only phonons with the periodicity of our supercell ( $\Gamma$-point phonons) were considered. The corresponding DOS for the models generated with the PBE functionals at $6.448 \mathrm{~g} / \mathrm{cm}^{3}$ and $5.75 \mathrm{~g} / \mathrm{cm}^{3}$ is reported in Ref. 15 to which we refer to for the sake of comparison. In 


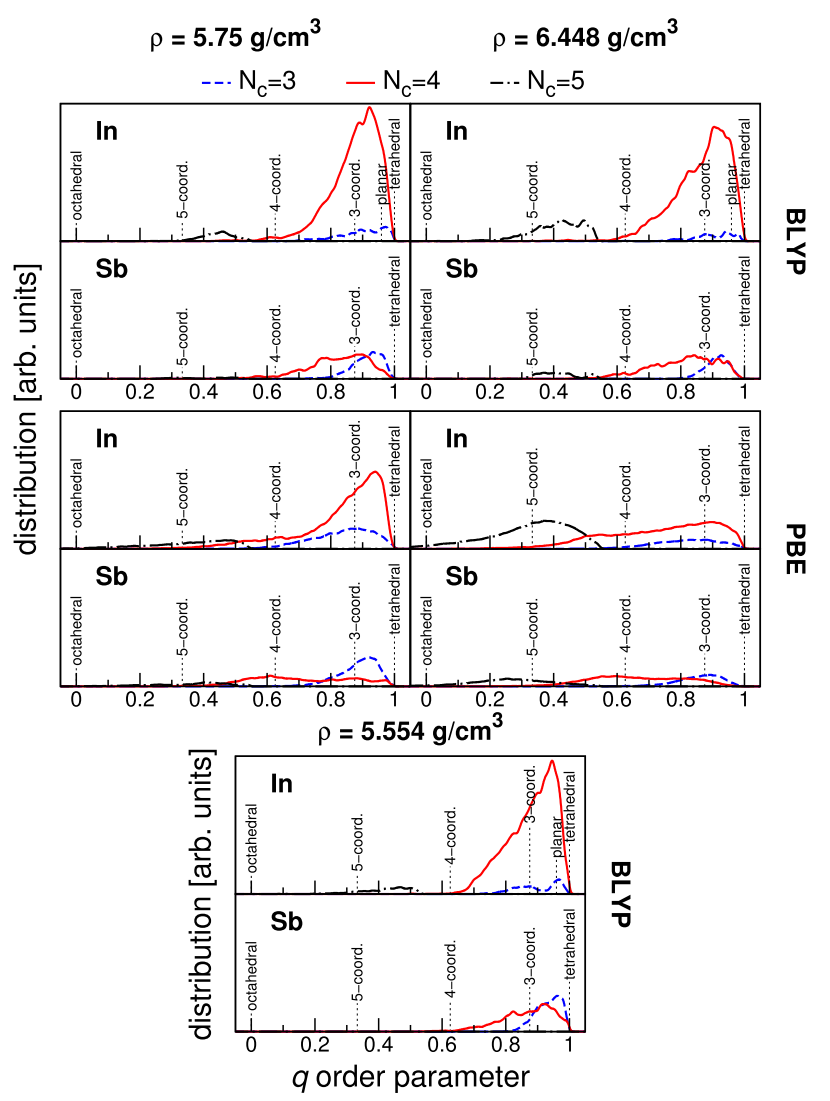

FIG. 11. Distribution of the local order parameter $q$ for tetrahedricity (see text) of a-In $\mathrm{SbTe}_{2}$ models generated with the BLYP (top panels) and PBE (central panels) functionals at the density of $5.75 \mathrm{~g} / \mathrm{cm}^{3}$ (left panel) or $6.448 \mathrm{~g} / \mathrm{cm}^{3}$ (right panel). The distribution for In, Sb, and Te atoms are given separately and are further resolved in the contributions from atoms with different coordination $\left(\mathrm{N}_{c}\right)$. Vertical dashed lines indicate the values of $q$ for selected ideal defective-octahedral geometries and for the tetrahedral geometry. The data for the BLYP model at the theoretical equilibrium density of $5.554 \mathrm{~g} / \mathrm{cm}^{3}$ are reported in the bottom panel.

Fig. 14 we also show the inverse participation ratio $(I P R)$ that quantifies the degree of localization of each phonon. The $I P R$ of the $j$ th vibrational mode is defined as

$$
I P R=\frac{\sum_{m}\left|\frac{\mathbf{e}(j, m)}{\sqrt{M_{m}}}\right|^{4}}{\left(\sum_{m} \frac{|\mathbf{e}(j, m)|^{2}}{M_{m}}\right)^{2}},
$$

TABLE IV. Statistics of the connectivity of tetrahedra for models a- $\mathrm{In}_{3} \mathrm{SbTe}_{2}$ generated with the BLYP and PBE functionals at the density of $5.75 \mathrm{~g} / \mathrm{cm}^{3}$, $6.448 \mathrm{~g} / \mathrm{cm}^{3}$, and $5.554 \mathrm{~g} / \mathrm{cm}^{3}$. The absolute numbers of tetrahedra of the different types are give in parenthesis.

\begin{tabular}{lccc}
\hline \hline \multicolumn{4}{c}{ Tetrahedra statistics } \\
\hline Model & Isolated & Corner-sharing & Edge-sharing \\
\hline BLYP $5.554 \mathrm{~g} / \mathrm{cm}^{3}$ & $\ldots$ & $84 \%(91)$ & $16 \%(17)$ \\
BLYP $5.75 \mathrm{~g} / \mathrm{cm}^{3}$ & $1 \%(1)$ & $71 \%(82)$ & $28 \%(33)$ \\
PBE $5.75 \mathrm{~g} / \mathrm{cm}^{3}$ & $1 \%(1)$ & $68 \%(48)$ & $31 \%(22)$ \\
BLYP $6.448 \mathrm{~g} / \mathrm{cm}^{3}$ & $\ldots$ & $94 \%(99)$ & $6 \%(6)$ \\
PBE $6.448 \mathrm{~g} / \mathrm{cm}^{3}$ & $12 \%(4)$ & $82 \%(28)$ & $6 \%(2)$ \\
\hline \hline
\end{tabular}

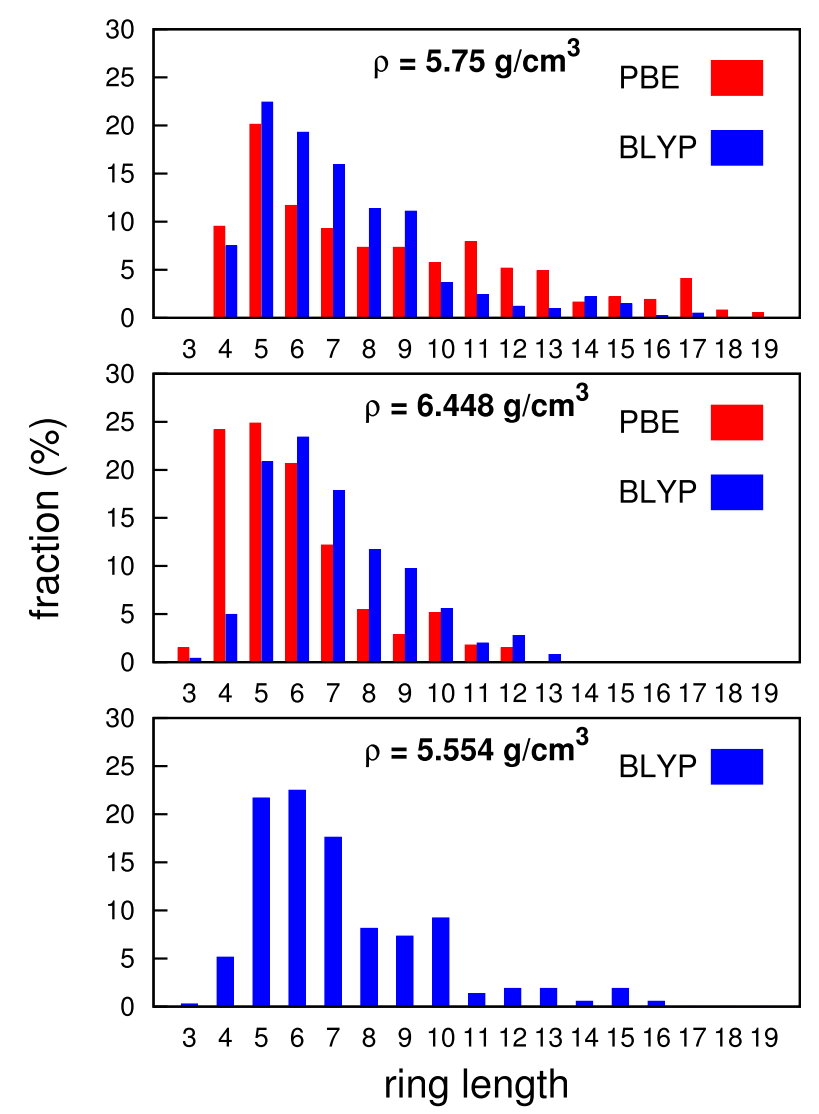

FIG. 12. Ring statistics of a- $\mathrm{In}_{3} \mathrm{SbTe}_{2}$ models generated with the BLYP and PBE functionals at the density of $5.75 \mathrm{~g} / \mathrm{cm}^{3}$ (top panel), $6.448 \mathrm{~g} / \mathrm{cm}^{3}$ (central panel), and $5.554 \mathrm{~g} / \mathrm{cm}^{3}$ (bottom panel).

where $\mathbf{e}(j, m)$ are phonon eigenvectors, while the sum over $m$ runs over the $N$ atoms in the unit cell with masses $M_{m}$. According to this definition, the value of the IPR varies from $1 / N$ for a completely delocalized phonon, to one for a mode completely localized on a single atom. Phonons above $150 \mathrm{~cm}^{-1}$ are mostly localized on tetrahedra centered on In. Note that due to the increased fraction of tetrahedra, the phonon DOS extends to higher frequencies with respect to

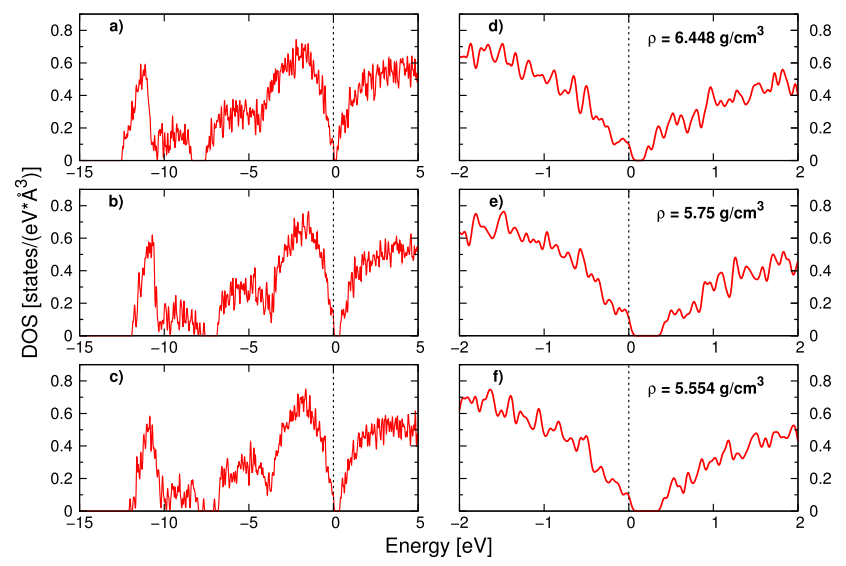

FIG. 13. Electronic density of states (BLYP functional) for the models of a$\mathrm{In}_{3} \mathrm{SbTe}_{2}$ at density of (a) $6.448 \mathrm{~g} / \mathrm{cm}^{3}$, (b) $5.75 \mathrm{~g} / \mathrm{cm}^{3}$, and (c) $5.554 \mathrm{~g} / \mathrm{cm}^{3}$. The KS energies are broadened by Gaussian functions of $27 \mathrm{meV}$ width. The zero of energy corresponds to the top of the valence band. (d)-(f) A zooming of the DOS of panels (a)-(c) close to the band gap. 

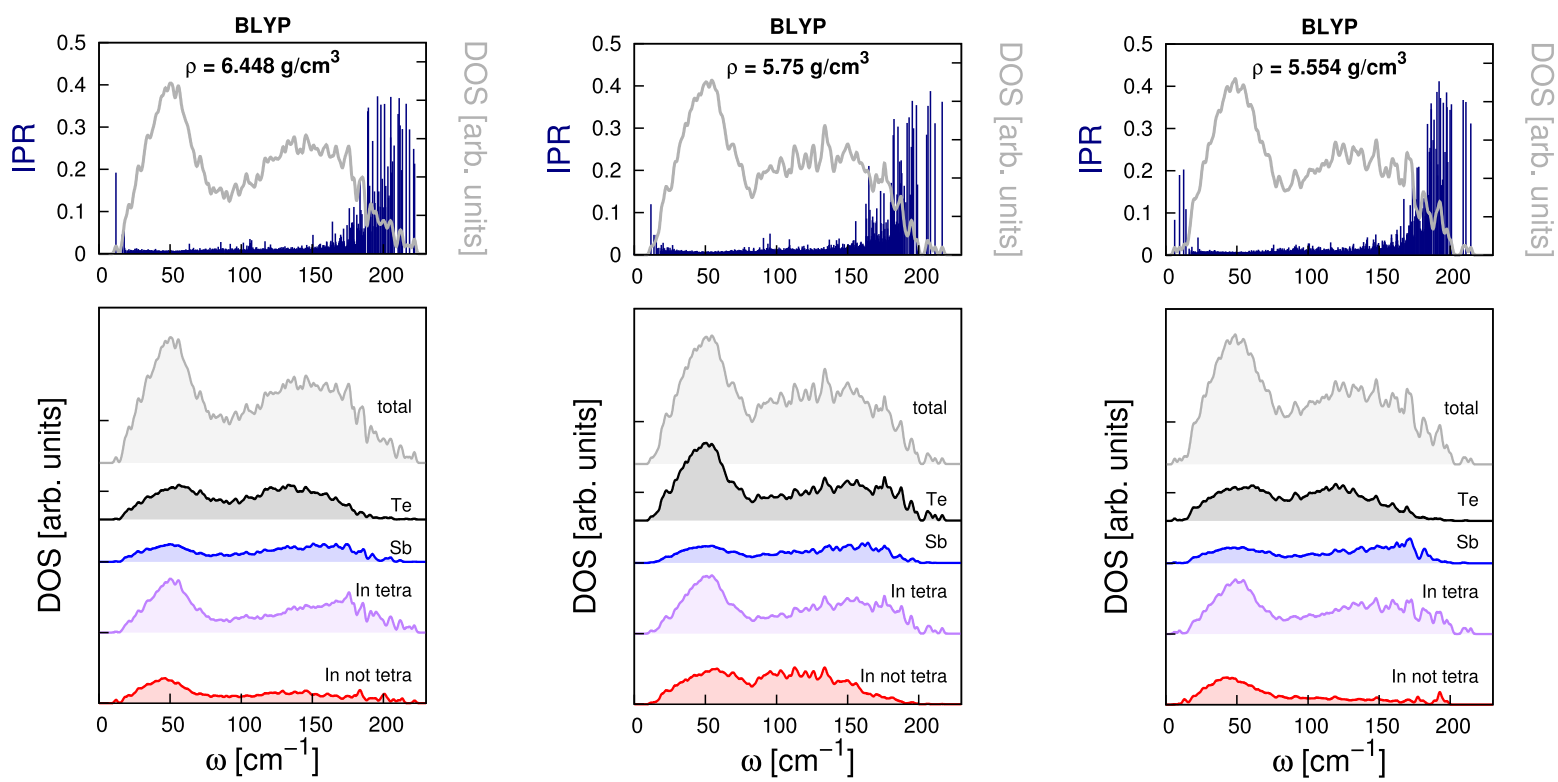

FIG. 14. Theoretical phonon DOS of the BLYP models of a- $\mathrm{In}_{3} \mathrm{Sb}_{1} \mathrm{Te}_{2}$ at density of $6.448 \mathrm{~g} / \mathrm{cm}^{3}$ (left panels), $5.75 \mathrm{~g} / \mathrm{cm}^{3}$ (central panels), and $5.554 \mathrm{~g} / \mathrm{cm}^{3}$ (right panels). The phonon IPR (blue spikes, see text for definition) is superimposed to the DOS in the upper panels. Projections on different species (Sb, Te, tetrahedral In, and non-tetrahedral In) are reported in the lower panels.

those of the more octahedral models generated with the PBE functional and reported in Ref. 15.

From the projected DOS we also computed the DebyeWaller factor for each species defined by ${ }^{63}$

$$
B_{\kappa}=\frac{8 \pi^{2}}{3}\left\langle\mathbf{u}_{\kappa}^{2}\right\rangle
$$

where $\kappa$ runs over the three species and $\left\langle\mathbf{u}_{\kappa}^{2}\right\rangle$ is the mean average square displacement of atoms of species $\kappa$ computed from harmonic phonons as

$$
\left\langle\mathbf{u}_{\kappa}^{2}\right\rangle=\sum_{j, m} \frac{\hbar}{\omega_{j}} \frac{|\mathbf{e}(j, m)|^{2}}{M_{\kappa}}\left[n_{B}\left(\frac{\hbar \omega_{j}}{k_{B} T}\right)+\frac{1}{2}\right],
$$

where $m$ runs over atoms of species $\kappa$, and $\omega_{j}$ is frequency of the $j$ th harmonic phonon. The temperature dependence is introduced by the Bose factor $n_{B}$.

The resulting Debye-Waller factors as a function of temperature are reported in Fig. 15; they are sizably lower than the values reported previously for the PBE models ${ }^{15}$ because of the overall blueshift of the phonon frequencies in the BLYP models due to higher fraction of tetrahedra.

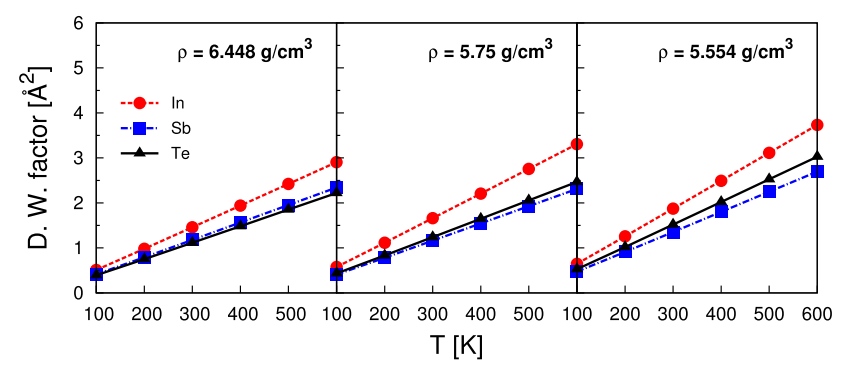

FIG. 15. Debye-Waller factor $\boldsymbol{B}_{\boldsymbol{K}}$ defined by Eq. (2) of In, Sb and Te atoms as a function of temperature in the BLYP models of a- $\mathrm{In}_{3} \mathrm{SbTe}_{3}$ at the three densities.
The calculated Debye-Waller factor averaged over the three species at $300 \mathrm{~K}$ is about $1.67 \AA^{2}$ in the model at $5.554 \mathrm{~g} / \mathrm{cm}^{3}$ which is very close to the value of $1.52 \AA^{2}$ measured experimentally from the Rietveld analysis of the crystalline cubic phase of $\mathrm{In}_{3} \mathrm{SbTe}_{2}$ in Ref. 42. However, we must consider that the latter experimental value includes the static displacement with respect to the ideal rocksalt geometry due to disorder. The theoretical thermal mean square displacement in the amorphous phase is therefore larger than the experimental value in the crystalline phase. The average Debye-Waller $B$ factor at $300 \mathrm{~K}$ of amorphous $\operatorname{In}_{3} \mathrm{SbTe}_{2}$ is also close to the corresponding theoretical value of about $2.0 \AA^{2}$ obtained for $\mathrm{Ge}_{2} \mathrm{Sb}_{2} \mathrm{Te}_{5}$ amorphous models in Ref. 47.

\section{CONCLUSIONS}

We have investigated the dependence of the structural and vibrational properties of models of amorphous $\mathrm{In}_{3} \mathrm{SbTe}_{2}$ on the choice of the exchange and correlation functional. Models of this phase change alloy generated by quenching from the melt with the BLYP functional have been compared with models generated with the most commonly used PBE functional in Ref. 15. As a preliminary investigation we have also compared PBE and BLYP models of the amorphous phase of the binary InSb compound. In both the InSb and $\mathrm{In}_{3} \mathrm{SbTe}_{2}$ compounds, the stronger electronic localization associated to the BLYP functional leads to a much larger fraction of tetrahedral configurations with respect to the PBE models that feature instead mostly defective octahedral or pyramidal bonding geometries. This outcome is similar to what has been documented for $\mathrm{GeSe}_{x}$ alloys, ${ }^{27,28}$ but it is at odds with the behaviour of $\mathrm{Ge}_{2} \mathrm{Sb}_{2} \mathrm{Te}_{5}$ for which very similar amorphous models have been generated with the PBE and BLYP functionals. ${ }^{29}$ The a- $\mathrm{In}_{3} \mathrm{SbTe}_{2}$ alloy appears as a mixture of the binary compounds InSb and InTe with very few $\mathrm{Sb}-\mathrm{Te}$ bonds as already discussed for the PBE models 
in Ref. 15. The BLYP models present, however, a mostly tetrahedral network whose structural features are more weakly dependent on the density than previously proposed on the basis of the PBE results. ${ }^{15}$ The strong dependence of the fraction of In and $\mathrm{Sb}$ in tetrahedral configurations on the choice of the exchange and correlation functional originates from the closeness in energy of the octahedral configurations. Indeed, $\mathrm{a}-\mathrm{InSb}$ is known to crystallize in the zincblend phase at normal conditions and in the rocksalt phase at a moderate pressure of 1-1.3 GPa. ${ }^{55}$ It actually turns out that the BLYP functional favors more strongly the zincblend phase with respect to the rocksalt one in crystalline InSb, the difference between the two phases at their equilibrium density at zero temperature being about $0.548 \mathrm{eV} /$ atom with the BLYP and $0.231 \mathrm{eV} /$ atom with the PBE functional.

Overall, the quest for an optimal description of bonding in Te-based amorphous phase change alloys seems to deserve further investigations for instance with other functionals such as hybrid functionals ${ }^{62}$ or functionals with non-local van der Waals corrections. ${ }^{24}$

\section{ACKNOWLEDGMENTS}

J.L. and T.D.K. would like to acknowledge financial support form the IDEE project of the Carl-Zeiss Foundation and the Graduate School of Excellence MAINZ. S.G. and M.B. acknowledge funding from the European Union Seventh Framework Programme No. FP7/2007-2013 under Grant Agreement No. 310339.

${ }^{1}$ M. Wuttig and N. Yamada, Nat. Mater. 6, 824 (2007).

${ }^{2}$ D. Lencer, M. Salinga, and M. Wuttig, Adv. Mater. 23, 2030 (2011).

${ }^{3}$ S. Raoux, W. Welnic, and D. Ielmini, Chem. Rev. 110, 240 (2010).

${ }^{4}$ A. Pirovano, A. L. Lacaita, A. Benvenuti, F. Pellizzer, and R. Bez, IEEE Trans. Electron Devices 51, 452 (2004).

${ }^{5}$ A. L. Lacaita and A. Redaelli, Microelectron. Eng. 109, 351 (2013).

${ }^{6}$ G. W. Burr et al., IBM J. Res. Dev. 22, 449 (2008).

${ }^{7}$ C. D. Wright, Y. Liu, K. I. Kohary, M. M. Aziz, and R. J. Hicken, Adv. Mater. 23, 3408 (2011); D. Kuzum, R. G. D. Jeyasingh, B. Lee, and H.-S. P. Wong, Nano Lett. 12, 2179 (2012).

${ }^{8}$ H. J. Shin, Y. S. Kang, A. Benayad, K. H. Kim, Y. M. Lee, M. C. Jung, T. Y. Lee, D. S. Suh, K. H. P. Kim, C. K. Kim, and Y. Khang, Appl. Phys. Lett. 93, 021905 (2008).

${ }^{9}$ Y. Maeda, H. Andoh, I. Ikuta, and H. Minemura, J. Appl. Phys. 64, 1715 (1988).

${ }^{10}$ E. T. Kim, J. Y. Lee, and Y. T. Kim, Phys. Status Solidi RRL 3, 103 (2009).

${ }^{11}$ Y. T. Kim, E. T. Kim, C. S. Kim, and J. Y. Lee, Phys. Status Solidi RRL 5, 98 (2011).

${ }^{12}$ R. Fallica, T. Stoycheva, C. Wiemer, and M. Longo, Phys. Status Solidi RRL 11, 1009 (2013).

${ }^{13}$ N. Papandreou, A. Pantazi, A. Sebastian, E. Eleftheriou, M. Breitwisch, C. Lam, and H. Pozidis, Solid-State Electron. 54, 991 (2010).

${ }^{14}$ K. Daly-Flynn and D. Strand, Jpn. J. Appl. Phys., Part 1 42, 795 (2003).

${ }^{15}$ J. H. Los, T. D. Kühne, S. Gabardi, and M. Bernasconi, Phys. Rev. B 88, 174203 (2013).

${ }^{16}$ R. O. Jones, Rev. Mod. Phys. 87, 897 (2015).

${ }^{17}$ S. Caravati, M. Bernasconi, T. D. Kühne, M. Krack, and M. Parrinello, Appl. Phys. Lett. 91, 171906 (2007).

${ }^{18}$ J. Akola and R. O. Jones, Phys. Rev. B 76, 235201 (2007).

${ }^{19}$ J. Hegedüs and S. R. Elliott, Nat. Mater. 7, 399 (2008).

${ }^{20}$ E. Spreafico, S. Caravati, and M. Bernasconi, Phys. Rev. B 83, 144205 (2011).

${ }^{21}$ J. P. Perdew, K. Burke, and M. Ernzerhof, Phys. Rev. Lett. 77, 3865 (1996).

${ }^{22}$ S. Grimme, J. Antony, S. Ehrlich, and H. Krieg, J. Chem. Phys. 132, 154104 (2010).
${ }^{23}$ M. Micoulaut, J. Chem. Phys. 133, 061103 (2013); M. Micoulaut, M.-V. Coulet, A. Piarristeguy, M. Johnson, C. J. Cuello, C. Bichara, H. FloresRuiz, and Pradel A, Phys. Rev. B 89, 174205 (2014); M. Micoulaut, K. Gunasekera, S. Ravindren, and P. Boolchand, ibid. 90, 094207 (2014).

${ }^{24}$ K. Lee, E. D. Murray, L. Kong, B. I. Lundqvist, and D. C. Langreth, Phys. Rev. B 82, 081101 (2010).

${ }^{25}$ J. Y. Raty, W. Zhang, J. Luckas, C. Chen, R. Mazzarello, C. Bichara, and M. Wuttig, Nat. Commun. 7, 7467 (2015).

${ }^{26}$ A. D. Becke, Phys. Rev. A 38, 3098 (1988); C. Lee, W. Yang, and R. G. Parr, Phys. Rev. B 37, 785 (1988).

${ }^{27}$ M. Micoulaut, R. Vuilleumier, and C. Massobrio, Phys. Rev. B 79, 214205 (2009).

${ }^{28}$ S. Le Roux, A. Bouzid, M. Boero, and C. Massobrio, J. Chem. Phys. 138, 174505 (2013).

${ }^{29}$ S. Caravati and M. Bernasconi, Phys. Status Solidi B 252, 261 (2015).

${ }^{30}$ K. Y. Kim, D.-Y. Cho, B.-Ki Cheong, D. Kim, H. Horii, and S. Han, J. Appl. Phys. 113, 134302 (2013).

${ }^{31}$ J. H. Los, T. D. Kühne, S. Gabardi, and M. Bernasconi, Phys. Rev. B 87, 184201 (2013).

${ }^{32}$ J. H. Los, S. Gabardi, M. Bernasconi, and T. D. Kühne, Comput. Mater. Sci. 117, 7 (2016).

${ }^{33}$ T. D. Kühne, M. Krack, F. R. Mohamed, and M. Parrinello, Phys. Rev. Lett. 98, 066401 (2007).

${ }^{34}$ R. Car and M. Parrinello, Phys. Rev. Lett. 55, 2471 (1985).

${ }^{35}$ T. D. Kühne, M. Krack, and M. Parrinello, J. Chem. Theory Comput. 5, 235 (2009).

${ }^{36}$ J. VandeVondele, M. Krack, F. Mohamed, M. Parrinello, T. Chassaing, and J. Hutter, Comput. Phys. Commun. 167, 103 (2005).

${ }^{37}$ M. Krack and M. Parrinello, in High Performance Computing in Chemistry NIC, edited by J. Grotendorst (FZ Jülich, 2004), Vol. 25, pp. 29-51; www.cp2k.org.

${ }^{38}$ S. Gödecker, M. Teter, and J. Hutter, Phys. Rev. B 54, 1703 (1996); M. Krack, Theor. Chem. Acc. 114, 145 (2005).

${ }^{39}$ J. P. Rino, D. S. Borges, and S. C. Costa, J. Non-Cryst. Solids 348, 17 (2004).

${ }^{40}$ O. Madelung, Semiconductors: Data Handbook (Springer Verlag, Berlin, 2004), pp. 154-161.

${ }^{41}$ K. Deneke and A. Rabenau, Z. Anorg. Allg. Chem. 333, 201 (1964).

${ }^{42}$ T. Schröder, T. Rosenthal, S. Grott, C. Stiewe, J. de Boor, and O. Oeckler, Z. Anorg. Allg. Chem. 639, 2536 (2013).

${ }^{43}$ J. H. Los and T. D. Kühne, Phys. Rev. B 87, 214202 (2013).

${ }^{44}$ R. L. McGreevy and L. Pusztai, Mol. Simul. 1, 359 (1988).

${ }^{45}$ R. L. McGreevy, J. Phys.: Condens. Matter 13, R877 (2001).

${ }^{46}$ N. J. Shevchik and W. Paul, J. Non-Cryst. Solids 16, 55 (1974).

${ }^{47}$ S. Caravati, M. Bernasconi, T. D. Kühne, M. Krack, and M. Parrinello, J. Phys.: Condens. Matter 21, 255501 (2009); errata, 21, 499803 (2009); errata, 22, 399801 (2010).

${ }^{48}$ S. Caravati, M. Bernasconi, and M. Parrinello, J. Phys.: Condens. Matter 22, 315801 (2010).

${ }^{49}$ S. Gabardi, S. Caravati, M. Bernasconi, and M. Parrinello, J. Phys.: Condens. Matter 24, 385803 (2012).

${ }^{50}$ R. Mazzarello, S. Caravati, S. Angioletti-Uberti, M. Bernasconi, and M. Parrinello, Phys. Rev. Lett. 104, 085503 (2010); 107, 039902(E) (2011).

${ }^{51}$ A. Bouzid and C. Massobrio, J. Chem. Phys. 137, 046101 (2012).

${ }^{52}$ S. Caravati, D. Colleoni, R. Mazzarello, T. D. Kühne, M. Krack, M. Bernasconi, and M. Parrinello, J. Phys.: Condens. Matter 23, 265801 (2011).

${ }^{53}$ G. C. Sosso, S. Caravati, R. Mazzarello, and M. Bernasconi, Phys. Rev. B 83, 134201 (2011).

${ }^{54}$ S. Caravati, M. Bernasconi, and M. Parrinello, Phys. Rev. B 81, 014201 (2010).

${ }^{55}$ O. Shimomurka, A. Saumin, N. Sakai, and S. Minomura, Philos. Mag. 34, 839 (1976).

${ }^{56}$ J. R. Errington and P. G. Debenedetti, Nature 409, 318 (2001).

${ }^{57}$ D. S. Franzblau, Phys. Rev. B 44, 4925 (1991).

${ }^{58}$ H. J. Monkhorst and J. D. Pack, Phys. Rev. B 13, 5188 (1976).

${ }^{59}$ P. Giannozzi et al., J. Phys.: Condens. Matter 21, 395502 (2009), http:// www.quantum-espresso.org.

${ }^{60}$ V. Deringer, W. Zhang, P. Rausch, R. Mazzarello, R. Dronskowski, and M. Wuttig, J. Mater. Chem. C 3, 9519 (2015).

${ }^{61}$ J. H. C. Hogg and H. H. Sutherland, Acta Cryst. 32, 2689 (1976).

${ }^{62}$ A. V. Krukau, O. A. Vydrov, A. F. Izmaylov, and G. E. Scuseria, J. Chem. Phys. 125, 224106 (2006).

${ }^{63}$ L.-M. Peng, S. L. Dudarev, and M. J. Whelan, High Energy Electron Diffraction and Microscopy (Oxford University Press, Oxford, 2004).

${ }^{64}$ See supplementary material at http://dx.doi.org/10.1063/1.4950817 for further details on the structural properties of $\mathrm{InSb}$ and $\mathrm{In}_{3} \mathrm{SbTe}_{2}$. 